\title{
Surgeon-Performed Ultrasound as Preoperative Localization Study in Patients with Primary Hyperparathyroidism
}

\author{
T.M. van Ginhoven ${ }^{a, b} \quad$ A.N. Morks ${ }^{a, c} \quad$ T. Schepers ${ }^{a, b} \quad$ P.W. de Graaf ${ }^{a} \quad$ P.C. Smit ${ }^{a}$ \\ ${ }^{\text {a }}$ Reinier de Graaf Groep, Department of Surgery, Delft, ${ }^{\text {b }}$ Department of Surgery, Erasmus MC, Rotterdam, and \\ 'Department of Surgery, UMCG, Groningen, The Netherlands
}

\section{Key Words}

Minimally invasive approach - Parathyroid hormone •

Primary hyperparathyroidism $\cdot$ Ultrasound

\begin{abstract}
Background: Minimally invasive parathyroidectomy is the treatment of choice for single-gland primary hyperparathyroidism. However, the exact location of the abnormal gland has to be established. Sestamibi scintigraphy, computed tomography and ultrasound (US) are commonly used modalities. We describe our experience in a non-academic center with surgeon-performed US (S-US) of the neck as preoperative localization study in patients with primary hyperparathyroidism (PHPT). Methods: Patients with a biochemically proven diagnosis of PHPT and preoperative S-US were included. Data were recorded prospectively. Perioperative gland location was compared to the preoperative S-US to determine sensitivity, specificity and accuracy rates. Results: Two of the 50 patients who underwent S-US were not subjected to surgery. In $85 \%$ of the patients analyzed by S-US, the appropriate abnormal gland(s) were identified. In $11 \%$, no gland was identified, but abnormal glands were found during surgery. Sensitivity of S-US in our hospital is $85 \%$, with a positive predictive value of $97 \%$. Conclusions: We achieved a satisfactory sensitivity rate. S-US provides anatomic infor-
\end{abstract}

mation to the surgeon which enables a more detailed operation planning, and it is a valuable diagnostic modality for patients with PHPT in our opinion. We hope that our data encourage other centers to implement this technique as well.

Copyright $\odot 2011$ S. Karger AG, Basel

\section{Introduction}

Primary hyperparathyroidism (PHPT) is most often due to one parathyroid adenoma secreting excessive parathyroid hormone (PTH) [1]. These patients are characterized by a persistent hypercalcemia with a concomitant increase or inappropriately high level of serum PTH. The standard of care for PHPT is surgical removal of all hyperfunctioning parathyroid tissue. In experienced hands, parathyroidectomy by conventional neck exploration (CNE) is successful in $95-99 \%$, with a very low complication rate $[2,3]$. However, $>90 \%$ of the patients have only one abnormal parathyroid gland, which makes it suitable for a focused approach (minimally invasive parathyroidectomy, MIP). Large studies have compared CNE with the minimally invasive approach (MIA) showing equal cure and complication rates [4-7]. The latter has the potential to be more cost effective with

\section{KARGER}

Fax +41613061234 E-Mail karger@karger.ch www.karger.com

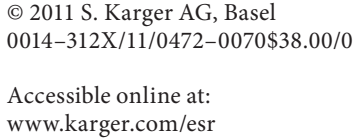

P.C. Smit, $\mathrm{MD}, \mathrm{PhD}$

Department of Surgery, Reinier de Graaf Gasthuis

Reinier de Graafweg 3-11

NL-2625 AD Delft (The Netherlands)

Tel. +31 152603060 , E-Mail smit@ rdgg.nl 
a shorter operation time and a shorter hospitalization [8]. Nowadays, MIP is the standard of care in case of uniglandular PHPT with preoperatively localized disease. Once the biochemical diagnosis of PHPT is established, preoperative localization of the abnormal glands is the next step towards MIP. Various imaging modalities can be used to identify the diseased glands. Technetium $99 \mathrm{~m}$-sestamibi scintigraphy (MIBI), which is commonly used, is based on a difference in absorption of sestamibi between normal and hyperfunctioning glands. The accuracy of MIBI depends on the particular scanning technique used [9], and reported sensitivity rates range from 54 to $88 \%[10,11]$. Sestamibi scintigraphy is reproducible and investigator independent, and abnormal parathyroid glands are relatively easy to detect. The scan does not allow precise anatomical localization, and only roughly indicates at which side and level of the neck the lesion is situated.

Computed tomography (CT) provides high-quality two (sometimes three)-dimensional anatomical images and has a reported sensitivity of $66 \%$ and specificity of $89 \%$ [12]. It is possible to use positron emission tomography in combination with CT to detect the hyperfunctioning gland, but this is not yet widely implemented [13]. Magnetic resonance imaging is another possible imaging modality with a reported sensitivity rate of $43.4-57.1 \%$ $[14,15]$. Ultrasound (US) is quick, non-invasive and does not require the use of radioactive materials. Unfortunately, US is highly operator dependent [16] and much information is lost when the two-dimensional images are stored. US performed by radiologists (R-US) have reported sensitivity rates of $59-96 \%$ [11, 17]. An increasing number of surgeons are using US as an extension of the clinical examination (surgeon-performed US, S-US). The use of office-based S-US to localize abnormal parathyroid glands preoperatively is not new. In several published studies, sensitivity rates of $66-76 \%$ have been reported [18-20].

The majority of data on S-US published in the last years were collected from university hospitals and referral centers. Results from non-academic hospitals remain scarce, which gives the impression that S-US is a modality which is mainly performed in an academic setting. Whether S-US measurements are useful in a non-academic hospital setting, usually treating a lower number of patients, remains obscure. The aim of this study is to describe the experience in performing S-US as an additional preoperative localization study in patients with PHPT in a non-academic center.

Surgeon-Performed Ultrasound for Primary Hyperparathyroidism

\section{Materials and Methods}

Data were collected at the Reinier de Graaf Hospital from August 2004 till September 2008. Before 2005, medical records of the participating patients were retrieved using operation-specific codes and relevant hospital coding systems. Thereafter, results were collected prospectively. All patients with a biochemically proven diagnosis of PHPT who were scheduled to undergo surgery underwent a preoperative US performed by the dedicated surgeon and were included in the analysis. Baseline characteristics (gender, age and preoperative laboratory values) were collected. All patients received preoperative localization studies consisting of MIBI and/or US investigation (R-US and/or S-US) and/or CT. MIBI and CT scans were performed using similar settings (slice thickness, timing and amount of radioactive material) during the whole study period.

\section{Surgeon-Performed Ultrasonography}

One of our endocrine surgeons (P.C.S.) performs neck US (Philips Envisor HD) at the outpatient clinic since 2004. No S-US were performed in the operating room just prior to surgery. The linear ray probe with a frequency of $3-12 \mathrm{MHz}$ is used with the software set on thyroid modus. The patient is examined in supine position with the neck in hyperextension. US provides an image of the entire neck, from the angles of the jaw to the sternal notch. When the probe is aimed downwards, the upper mediastinum can be visualized. However, the retrocervical tracheal space cannot be studied by ultrasound as the air in the trachea hinders the passage of the ultrasound waves. Previous neck surgery, radiation therapy and goiter negatively affect US. When a possible enlargement of the parathyroid gland was identified, color Doppler US was used to determine the vascularity of the lesion to identify a vascular pedicle strengthening the diagnosis of a parathyroid adenoma. Parathyroid adenomas were defined as any oval, elongated, or lobulated lesion connected with the thyroid gland during swallowing without a central hilum (characteristic for a lymph node). Lesions with the same characteristics but an increased and/or irregular reflection pattern, cystic changes and/or calcifications representing degeneration and measuring from $1-2$ to $\sim 5 \mathrm{~cm}$ in length were also considered to be an adenoma. When the solitary adenoma was located posterior to the thyroid lobe near the middle portion, it was considered an adenoma of the superior type. It was considered an inferior type when it was located near the lower pole of a thyroid lobe or inferior to it and having, at least in part, a close relationship with the anterior muscular wall of the neck. Any other location was considered aberrant. US takes approximately $10-15 \mathrm{~min}$, and findings are directly recorded in the patient's chart, with a detailed map (US pictures, drawings and text) for the scheduled surgical approach. The location of the future incision is described using the midline and the clavicula as landmarks.

\section{Surgery}

All procedures, both conventional neck explorations and MIP, were performed under general anesthesia by one of the two dedicated endocrine surgeons, either P.W.d.G. or P.C.S. P.C.S., who also performed S-US, carried out $>80 \%$ of the operations. The MIP, consisting of a small incision $(<3 \mathrm{~cm})$ above the suspect gland, was the operation of choice for single-gland disease. PTH levels were assessed intraoperatively to support the success of the minimally invasive procedure. After surgery, all normo- and hy- 
pocalcemic patients with normal PTH levels were considered cured. Persistent disease was defined as persistent hypercalcemia in the first 3 months after surgery. Recurrent disease was defined as hypercalcemia returning later than 3 months postoperatively. Serum calcium levels were obtained the evening after surgery, the next day and during visits at the outpatient clinic. These visits were planned approximately 2 weeks postoperatively at the Department of Surgery, and 3 and 12 months after surgery at the Department of Endocrinology. In case of temporary hypocalcemia, calcium supplementation was prescribed.

\section{Statistical Analysis}

Only descriptive statistics are used. Data were tested for normality using the Shapiro-Wilk test and visual assessment. Normally distributed data are presented as means \pm SD. Non-normally distributed data are reported as medians and ranges. For each imaging modality, any gland that was identified as abnormal parathyroid tissue by imaging. and confirmed as such by operative findings was scored as true positive (TP). A finding was false positive (FP) if operative findings did not confirm an abnormality of the gland detected by imaging. A finding was true negative (TN) if the gland was identified as normal by imaging and this was confirmed by intra-operative PTH levels and operative findings (among patients who underwent bilateral neck exploration). A finding was false negative (FN) if S-US failed to identify the abnormal gland but an abnormality was detected during the operation. Sensitivity was defined as $\mathrm{TP} /(\mathrm{TP}+\mathrm{FN})$, specificity was defined as $\mathrm{TN} /(\mathrm{TN}+\mathrm{FP})$, positive predictive value was defined as $\mathrm{TP} /(\mathrm{TP}+\mathrm{FP})$, and negative predictive value was defined as $\mathrm{TN} /(\mathrm{TN}+\mathrm{FN})$. The S-US localization was scored correct if the imaging technique predicted the proper quadrant (namely, upper left, upper right, lower left or lower right). The prediction was compared to the 'gold standard', which is for abnormal glands perioperative surgical findings combined with an abnormal gland during pathological analysis [abnormal glands include: adenomas (characterized by a neoplastic monoclonal process), hyperplastic glands (characterized by a polyclonal proliferation) or unsure (adenoma or hyperplasia)]. A cured patient indicates that there is no abnormal tissue left and all 'abnormal' glands have been removed, thus rendering the difference between adenoma and hyperplasia less important in this study. For normal glands, the prediction was compared to the pathological analysis or, if the gland remained in situ, a cured patient. Therefore, uncured patients are left out of this part of the analysis.

\section{Results}

From August 2004 until September 2008, 50 patients were included in the study. The analysis comprised 33 (66\%) women and 17 (34\%) men, with a median age of 58 years (range 20-82). The preoperative median serum calcium level was $2.79 \mathrm{mmol} / \mathrm{l}$ (range 2.56-3.26, normal range 2.20-2.60) and the median serum PTH level was $12.40 \mathrm{pmol} / \mathrm{l}$ (range 5.6-70.4, normal range 1.0-5.5). One patient had (high) normal calcium levels under calciumlowering medication $(\mathrm{PTH}=8.6)$. All other patients were hypercalcemic.

\section{Surgery}

In 2 patients, it was decided to refrain from surgery due to the mild character of the disease combined with high comorbidity of the patients. They were excluded from further analysis. Forty-four patients underwent a first surgical procedure for PHPT. The MIP was chosen in 38 patients (86\%); 6 patients (14\%) underwent CNE. Reasons for CNE were multiple gland disease, recurrent nerve paresis due to unknown etiology, unequivocal imaging results or no reason specified.

In 4 patients, only the second operations for persistent hypercalcemia (reoperation) are included in the study, as the first operations were performed prior to the start of our study. Two patients could be cured by means of MIP. The other 2 patients are discussed in more detail as they had persistent disease. One patient developed recurrent disease after surgery in 1979. The patient was operated by means of a targeted approach to the left lower parathyroid, based on the results of the sestamibi scan. As intraoperative PTH levels failed to decrease, the surgical procedure was converted to a conventional neck operation. No abnormal parathyroid glands were identified. This patient was referred to a tertiary center. The last communication reports that, based on low urinary calcium levels, the diagnosis of hypocalciuric hypercalcemia is considered. The other patient was also treated for recurrent disease years after her first operation. CNE revealed an adenoma which was removed from a location different to that detected by S-US. This patient has, at the time of reporting, a normal calcium level with a high PTH level. Regrettably, during follow-up, a malignancy with metastasis (non-parathyroid) was diagnosed in this patient and evaluation of the hyperparathyroidism was discontinued.

\section{Results of Surgeon-Performed Ultrasound}

In total, 50 US were performed by the surgeon at the outpatient department. Four patients were excluded from the following analysis as no definite conclusion can be made about all 4 parathyroid glands $(\mathrm{n}=2$, not operated; $\mathrm{n}=2$ not cured). Preoperative S-US identified all the important anatomical landmarks: namely trachea, esophagus (paraesophageal grove), thyroid gland, carotid artery and jugular vein in all patients. All patients underwent both MIBI scanning and S-US. A CT scan was performed in 20/46 (44\%) patients and R-US in 26/46 (57\%) patients. Results from MIBI, R-US and S-US are shown in table 1.

In 40 of the 46 (87\%) S-USs, the suspected lesions seen on S-US truly were hyperfunctioning glands, however in 2 cases multiple-gland disease was overlooked (4\%). In 1 case, multiple-gland disease was adequately predicted. In 
Table 1. Results of preoperative localization studies

\begin{tabular}{|c|c|c|c|c|c|c|c|c|c|c|}
\hline & \multicolumn{3}{|c|}{ Glands, n } & \multirow[t]{2}{*}{$\mathrm{TN}$} & \multirow[t]{2}{*}{$\mathrm{TP}$} & \multirow[t]{2}{*}{ SN } & \multirow[t]{2}{*}{ SP } & \multirow[t]{2}{*}{ PPV } & \multirow[t]{2}{*}{ NPV } & \multirow[t]{2}{*}{ Accuracy } \\
\hline & total & abnormal & normal & & & & & & & \\
\hline $\operatorname{MIBI}(\mathrm{n}=46)$ & 184 & 40 & 144 & 135 & 37 & $80 \%$ & $98 \%$ & $93 \%$ & $94 \%$ & $93 \%$ \\
\hline S-US $(n=46)$ & 184 & 42 & 142 & 135 & 41 & $85 \%$ & $99 \%$ & $98 \%$ & $95 \%$ & $96 \%$ \\
\hline R-US $(\mathrm{n}=26)$ & 104 & 14 & 90 & 76 & 13 & $48 \%$ & $99 \%$ & $93 \%$ & $84 \%$ & $86 \%$ \\
\hline
\end{tabular}

$\mathrm{TP}=$ True positive; $\mathrm{SN}=$ sensitivity; $\mathrm{TN}$ = true negative; $\mathrm{SP}=$ specificity; $\mathrm{PPV}=$ positive predictive value; $\mathrm{NPV}=$ negative predictive value. Numbers of patients are shown in parentheses.

5 cases (11\%), no gland was identified by S-US, while an abnormal gland was found during surgery. In 1 patient (2\%), S-US identified an abnormal gland, but the gland was in the wrong quadrant (adequate side of the neck).

\section{Follow-Up and Complications}

The mean calcium level of all cured patients (1-year follow-up) was $2.36 \mathrm{mmol} / \mathrm{l}$ (range 2.25-2.53, normal range 2.20-2.60). Two patients are not considered cured, one of them is currently being treated for a metastatic disease and the other is diagnosed with hypocalciuric hypercalcemia (both are described in the Surgery section).

Three of the 48 operated patients (6\%) had temporary postoperative hypocalcaemia for which 1 patient needed calcium supplementation. One patient had temporary hoarseness, most likely due to irritation by intubation, which restored rapidly. A re-operation for hemorrhage was needed in 1 patient.

\section{Discussion}

This study encompasses patients with PHPT who underwent preoperative S-US. The aim of our article is to describe the experience of US performed in a single nonacademic center by a surgeon. As surgeons become more familiar with the use of US, it is more often applied as an extension of the physical examination during the initial office visit [20]. This trend is not restricted to parathyroid disease, it is also observed in other fields of medicine [2123]. In our opinion, one of the main benefits of S-US is the support of sonographic findings prior to and during surgical treatment. The knowledge of the exact gland location enables the surgeon to perform a targeted tissue dissection, thereby possibly decreasing the risk of recurrent nerve injury and reducing scar formation. A prospective trial in a large cohort should be conducted to determine whether S-US leads to lower complication rates and shorter operation times.

Although it may require more logistical planning, it may be beneficial to perform S-US directly before surgery in the operating room. This enables the surgeon to directly plan the operation and the incision site, without the need for 'remembering' the case from the outpatient department. However, if no gland is found and more imaging is needed, the operation needs to be rescheduled. In addition, in our hospital, we use S-US as an additional imaging modality. We feel that if the S-US shows no abnormal glands, the other imaging modalities (MIBI, CT, R-US) must show an abnormal gland in order to proceed to MIP. Otherwise, CNE must be considered.

We acknowledge the fact that the number of patients in this study is limited and therefore these results should be interpreted with care. We also emphasize that it was not our intention to compare S-US with R-US, as our data were not suitable for this purpose. For example, S-US results cannot be compared with R-US results as the latter have been done by various radiologists, instead of one investigator. Secondly S-US is most of the times performed after the MIBI results were available, whereas some R-US were the first imaging modality applied.

Recent studies have shown US to be highly accurate in localizing parathyroid adenomas with reported sensitivity rates of up to $70-92.5 \%[17,19,24]$. Our sensitivity rate is $85 \%$.

The incidence of multiglandular disease in this study was $6 \%$, which is within the normal range, but in the lower region of the incidence rates reported in the literature $[18,25-27]$. This could be due to the relatively small numbers of patients in our study. As previously reported, preoperative localization is less accurate in multiglandular disease compared to single-gland disease [26]. The lower incidence of multiglandular disease in our study population may be a bias towards the results. 
Several studies suggest that if S-US shows one abnormal gland, no further examination/imaging is needed and one may perform MIP [19]. Our study is not suited to draw conclusions regarding this statement. Many S-US are performed while knowing the results of the MIBI scanning, thus introducing bias. Although we report test characteristics of our imaging modalities, we cannot directly compare them due to the aforementioned bias.

Altogether, we regard S-US as a unique opportunity for the surgeon to plan the operation in detail by means of the detailed anatomical information provided by S-US. Despite the limitations of US (multiglandular disease/interobserver variety), the benefits (non-invasive approach with detailed real-time anatomical information which is easy to use) have led us to use S-US in every patient visiting our outpatient clinic and achieve a very acceptable sensitivity rate. We recommend the use of S-US and hope that our data encourage other non-academic centers to apply S-US.

\section{References}

1 Sitges-Serra A, Bergenfelz A: Clinical update: sporadic primary hyperparathyroidism. Lancet 2007;370:468-470.

2 Lee JA, Inabnet WB 3rd: The surgeon's armamentarium to the surgical treatment of primary hyperparathyroidism. J Surg Oncol 2005;89:130-135

3 Ruda JM, Hollenbeak CS, Stack BC Jr: A systematic review of the diagnosis and treatment of primary hyperparathyroidism from 1995 to 2003. Otolaryngol Head Neck Surg 2005;132:359-372.

4 Bergenfelz A, Kanngiesser V, Zielke A, Nies C, Rothmund M: Conventional bilateral cervical exploration versus open minimally invasive parathyroidectomy under local anaesthesia for primary hyperparathyroidism. $\mathrm{Br}$ J Surg 2005;92:190-197.

5 Bergenfelz A, Lindblom P, Tibblin S, Westerdahl J: Unilateral versus bilateral neck exploration for primary hyperparathyroidism: a prospective randomized controlled trial. Ann Surg 2002;236:543-551.

6 Udelsman R: Six hundred fifty-six consecutive explorations for primary hyperparathyroidism. Ann Surg 2002;235:665-670, discussion 670-662.

7 Smit PC, Borel Rinkes IH, van Dalen A, van Vroonhoven TJ: Direct, minimally invasive adenomectomy for primary hyperparathyroidism: an alternative to conventional neck exploration? Ann Surg 2000;231:559-565.

8 Russell CF, Dolan SJ, Laird JD: Randomized clinical trial comparing scan-directed unilateral versus bilateral cervical exploration for primary hyperparathyroidism due to solitary adenoma. Br J Surg 2006;93:418421.

9 Sharma J, Mazzaglia P, Milas M, Berber E, Schuster DM, Halkar R, Siperstein A, Weber CJ: Radionuclide imaging for hyperparathyroidism (HPT): which is the best technetium-99m sestamibi modality? Surgery 2006;140:856-863, discussion 863-865.

10 Chapuis Y, Fulla Y, Bonnichon P, Tarla E, Abboud B, Pitre J, Richard B: Values of ultrasonography, sestamibi scintigraphy, and intraoperative measurement of 1-84 PTH for unilateral neck exploration of primary hy- perparathyroidism. World J Surg 1996;20: 835-839, discussion 839-840

11 Siperstein A, Berber E, Barbosa GF, Tsinberg M, Greene AB, Mitchell J, Milas M: Predicting the success of limited exploration for primary hyperparathyroidism using ultrasound, sestamibi, and intraoperative parathyroid hormone: analysis of 1,158 cases. Ann Surg 2008;248:420-428

12 Harari A, Zarnegar R, Lee J, Kazam E, Inabnet WB 3rd, Fahey TJ 3rd: Computed tomography can guide focused exploration in select patients with primary hyperparathyroidism and negative sestamibi scanning. Surgery 2008;144:970-976, discussion 976-979.

13 Hindie E, Ugur O, Fuster D, O’Doherty M Grassetto G, Urena P, Kettle A, Gulec SA, Pons F, Rubello D: 2009 EANM parathyroid guidelines. Eur J Nucl Med Mol Imaging 2009;36:1201-1216

14 Wakamatsu H, Noguchi S, Yamashita H, Yamashita $\mathrm{H}$, Tamura S, Jinnouchi S, Nagamachi S, Futami S: Technetium-99m tetrofosmin for parathyroid scintigraphy: a direct comparison with ${ }^{99 \mathrm{~m}} \mathrm{Tc}-\mathrm{MIBI},{ }^{201} \mathrm{Tl}$, MRI and US. Eur J Nucl Med 2001;28:1817-1827.

15 Wakamatsu H, Noguchi S, Yamashita H, Yamashita H, Tamura S, Jinnouchi S, Nagamachi S, Futami S: Parathyroid scintigraphy with ${ }^{99 \mathrm{~m}} \mathrm{Tc}-\mathrm{MIBI}$ and ${ }^{123} \mathrm{I}$ subtraction: a comparison with magnetic resonance imaging and ultrasonography. Nucl Med Commun 2003;24:755-762.

16 Reeder SB, Desser TS, Weigel RJ, Jeffrey RB: Sonography in primary hyperparathyroidism: review with emphasis on scanning technique. J Ultrasound Med 2002;21:539-552, quiz 553-534.

17 Abboud B, Sleilaty G, Rabaa L, Daher R, Abou Zeid H, Jabbour H, Hachem K, Smayra T: Ultrasonography: highly accuracy technique for preoperative localization of parathyroid adenoma. Laryngoscope 2008; 118 : 1574-1578.

18 Van Husen R, Kim LT: Accuracy of surgeonperformed ultrasound in parathyroid localization. World J Surg 2004;28:1122-1126.

19 Solorzano CC, Carneiro-Pla DM, Irvin GL 3rd: Surgeon-performed ultrasonography as the initial and only localizing study in sporadic primary hyperparathyroidism. J Am Coll Surg 2006;202:18-24.

20 Milas M, Stephen A, Berber E, Wagner K, Miskulin J, Siperstein A: Ultrasonography for the endocrine surgeon: a valuable clinical tool that enhances diagnostic and therapeutic outcomes. Surgery 2005;138:1193-1200, discussion 1200-1191.

21 Lindelius A, Torngren S, Pettersson H, Ada$\mathrm{mi}$ J: Role of surgeon-performed ultrasound on further management of patients with acute abdominal pain: a randomised controlled clinical trial. Emerg Med J 2009;26: 561-566.

22 Jabiev AA, Ikeda MH, Reis IM, Solorzano CC, Lew JI: Surgeon-performed ultrasound can predict differentiated thyroid cancer in patients with solitary thyroid nodules. Ann Surg Oncol 2009;16:3140-3145.

23 Copeland DR, Cosper GH, McMahon LE, Boneti C, Little DC, Dassinger MS, Kokoska ER, Jackson RJ, Smith SD: Return of the surgeon in the diagnosis of pyloric stenosis. J Pediatr Surg 2009;44:1189-1192, discussion 1192.

24 Haber RS, Kim CK, Inabnet WB: Ultrasonography for preoperative localization of enlarged parathyroid glands in primary hyperparathyroidism: comparison with ${ }^{99} \mathrm{~m}$ technetium sestamibi scintigraphy. Clin Endocrinol (Oxf) 2002;57:241-249.

25 Purcell GP, Dirbas FM, Jeffrey RB, Lane MJ, Desser T, McDougall IR, Weigel RJ: Parathyroid localization with high-resolution ultrasound and technetium Tc 99m sestamibi. Arch Surg 1999;134:824-828, discussion 828-830

26 Yeh MW, Barraclough BM, Sidhu SB, Sywak MS, Barraclough BH, Delbridge LW: Two hundred consecutive parathyroid ultrasound studies by a single clinician: the impact of experience. Endocr Pract 2006;12: 257-263.

27 Molinari AS, Irvin GL 3rd, Deriso GT, Bott $\mathrm{L}$ : Incidence of multiglandular disease in primary hyperparathyroidism determined by parathyroid hormone secretion. Surgery 1996;120:934-936, discussion 936-937. 